\title{
Mortality and excess risk in US adults with pre- diabetes and diabetes: a comparison of two nationally representative cohorts, 1988-2006
}

Andrew Stokes ${ }^{1 *}$ and Neil K Mehta ${ }^{2}$

\begin{abstract}
Background: There is strong evidence on the efficacy of behavioral modification and treatment for reducing diabetes incidence and diabetes-related morbidity and mortality in persons with pre-diabetes and diabetes. But the extent to which the evidence has translated into gains in health in these population sub-groups in the US is unclear. Monitoring national diabetes-related mortality levels over time is important for evaluating the effectiveness of the US health system response to diabetes.

Methods: We identified individuals with pre-diabetes and diabetes using Hemoglobin A1c. Two consecutive periods for investigating differences in mortality according to categories of glycemia were derived using nationally representative survey data on US adults ages 35-74 from subsequent rounds of the National Health and Nutrition Examination Survey (1988-1994 and 1999-2002). Age-standardized mortality rates were calculated for individuals with pre-diabetes and diabetes and proportional hazards models were used to assess change in the relative risks of dysglycemia (pre-diabetes and diabetes) adjusting for multiple confounding factors.

Results: Age-standardized mortality rates in individuals with pre-diabetes and diabetes showed no statistically significant change between 1988-2001 and 1999-2006. In individuals with pre-diabetes, mortality rates were 11.19 and 14.02 deaths per 1,000 person-years in the early and later period, respectively. The corresponding values for individuals with diabetes were 20.34 and 20.82 deaths per 1,000 person-years. In contrast, the absolute level of mortality in the normo-glycemic population declined significantly between 1988-2001 and 1999-2006 (7.81 to 6.04; $\mathrm{p}$ for difference $<0.05$ ). Adjusting for social and demographic variables, smoking and body mass index in a multivariate analysis, the hazard ratio of dysglycemia increased from 1.62 (95\% Cl: 1.36-1.93) in 1988-2001 to 2.36 (95\% Cl: 1.70-3.27) in 1999-2006 ( $p$ for difference < 0.05).
\end{abstract}

Conclusions: We find no evidence of declines in excess mortality in persons with dysglycemia between 1988-2001 and 1999-2006, a result that was robust to adjustment for social and demographic variables, smoking and body mass index. In the context of long-term secular declines in mortality in the US population, our findings suggest that individuals with pre-diabetes and diabetes should be an important focus of future interventions aimed at improving population health in the US.

Keywords: Pre-diabetes, Diabetes, Dysglycemia, Diabetes management, Mortality, Mortality change

\footnotetext{
* Correspondence: astokes@sas.upenn.edu

${ }^{1}$ Population Studies Center, University of Pennsylvania, 3718 Locust Walk, 239

McNeil Building, Philadelphia, PA 19104-6298, USA

Full list of author information is available at the end of the article
} 


\section{Background}

Diabetes mellitus is a leading cause of death in the US [1]. Clinical trial data have demonstrated the efficacy of lifestyle and pharmacological interventions for reducing the incidence and complications of diabetes [2-5]. Concurrent management of cardiovascular risk factors in individuals with diabetes has shown to result in particularly large improvements in health outcomes [6,7].

Despite the demonstrated efficacy of primary and secondary diabetes prevention strategies, achieving improved population health outcomes in practice requires aggressive screening and overcoming the complexities of managing diabetes and associated conditions. Diabetes management requires long-term medical care, input and coordination across health providers, patient self-education and motivation and addressing multiple behavioral, metabolic and cardiovascular risk factors simultaneously [8]. Previous studies have used diabetes as an indicator of health systems performance in recognition that successful implementation of these strategies relies centrally on high quality and coordinated health care [9].

Evidence on the processes of care for individuals with diagnosed diabetes for the 1990s and early 2000s indicates that treatment rates have improved, but that cardiovascular risk factor control remains sub-standard [10-12]. With respect to glycemic control, little to no change was observed over this period $[11,12]$. Information on mortality trends in individuals with pre-diabetes and diabetes is critical for a full assessment of progress towards the more effective control of diabetes-related outcomes in the US population. Trends have been assessed in three previous nationally representative studies of the US population [13-15]. In all of the previous studies, individuals with diabetes were identified using self-reported data on having ever received a diagnosis of diabetes by a health care provider. However, diagnostic criteria [16] and levels of screening $[17,18]$ have changed over time, affecting selection into the population with diagnosed diabetes and complicating interpretation of trends. Also, because diabetes is undiagnosed in a third to a fifth of US adults with the condition depending on the criterion used [19], trends identified in prior studies may not reflect trends occurring among the overall diabetic population. Information on trends in the overall population with diabetes is important for a more complete assessment of the health system response to diabetes, the success of which depends both on diagnosis and control of diabetes.

In this study, we investigate change over time in absolute and excess mortality rates in individuals with prediabetes and diabetes (collectively, 'dysglycemia') during the period 1988-2006, which is the most recent period for which nationally representative data are available.
We extend the focus to individuals with pre-diabetes as this group is at high risk for developing diabetes and its complications and is considered a high-priority group for intervention [8]. We use a clinical diagnostic measure, Hemoglobin A1c (HbA1c), to identify individuals with dysglycemia and adjust for key confounding factors in a multivariate analysis of mortality change.

\section{Methods}

We established two consecutive periods for investigating mortality change according to diabetes status using data from the National Health and Nutrition Examination Survey (NHANES). NHANES is a series of nationally representative surveys of the non-institutionalized U.S. population conducted by the National Center for Health Statistics (NCHS). The survey includes an examination component in which extensive medical data are collected by trained nurses in mobile clinics or in homevisits and is linked to the National Death Index, a computerized index of death record information maintained by the NCHS [20]. The earlier period in our analysis was constructed using data from 1988-1994 (NHANES III) and the later period using data from 1999-2002 (NHANES CTS). To reduce bias that may result from differential follow-up across the two mortality periods, we restricted mortality follow-up in NHANES III to 7.7 years, the maximum follow-up available in NHANES CTS. The resulting periods for our analysis of mortality change were 1988-2001 for NHANES III and 1999-2006 for NHANES CTS.

We restricted the sample to individuals ages 35-74 who underwent examination and had non-missing information on diabetes and death $(3.7 \%$ and $0.1 \%$ of persons were missing information on each of these items). The resulting sample included 9,210 observations on the earlier cohort and 5,470 observations on the later cohort. We defined diabetes on the basis of the HbA1c test, which is standardized to the methods of the Diabetes Control and Complications Trial in both periods [21]. A distinct advantage of HbA1c is that it reflects average glycaemia over a prolonged period of time and exhibits greater pre-analytic stability and lower intraindividual variation than other diagnostic markers, such as fasting plasma glucose (FPG) [22]. We used the most recent national American Diabetes Association (ADA) guidelines [8] to classify individuals into categories of non-diabetes (HbA1c less than 5.7\%), pre-diabetes (HbA1c between $5.7 \%$ and $6.5 \%$ ) or diabetes (HbA1c more than $6.5 \%$ ). We further classified as diabetic persons whose $\mathrm{HbA1c}$ values were below $6.5 \%$ but who reported use of an oral hypoglycemic agent or insulin. When referring collectively to individuals with prediabetes and diabetes, we use the term 'dysglycemia'. To minimize bias, we did not consider information on 
physician-diagnosed diabetes because diagnostic criteria changed over the period of analysis [23]. Approximately $1 \%$ of normo-glycemic individuals not currently taking medications for diabetes reported a previous diagnosis of the condition.

We calculated age-standardized mortality rates separately by diabetes status and period as the ratio of deaths to person-years of follow-up, in each case using the year 2000 Census population as the standard. Within each period, we calculated relative risks comparing prediabetes and diabetes categories to no diabetes.

We also implemented Cox proportional hazards models to estimate mortality risks associated with prediabetes and diabetes adjusted for covariates and to test for the significance of differences in these risks across periods. Attained age was used as analysis time. We imputed missing covariate values using multiple imputation with Amelia II software [24,25]. The imputation model included all model covariates, including transformations. Overall, $1.1 \%$ of persons were missing information on BMI, $0.5 \%$ on education and $0.1 \%$ on smoking. Changes in the hazard ratios were estimated by introducing an interaction into the model between diabetes status and an indicator variable for period. We initially assessed whether the proportionate change in the hazard ratios differed across the two dysglycemic groups. The two values were statistically indistinguishable from one another; therefore in subsequent analysis we pooled individuals with pre-diabetes and diabetes into a dysglycemic group. We additionally examined sex-specific differences in the interaction, but found no statistical differences by sex so we present results for the sexes combined. In Model 1, we adjusted for social and demographic covariates, including sex, race/ethnicity (indicators for non-Hispanic black and Hispanic ethnicity) and education (less than high-school education, high-school education and more than high-school education). In Model 2, we additionally adjusted for smoking using categories of current, former and never smoker. In Model 3, we extended Model 2 by including linear and quadratic terms for body mass index (BMI) [26]. We adjusted for smoking and BMI as they are strong correlates of diabetes and previous research suggests that smoking cessation may increase the risks of developing diabetes [27,28].

We introduced interaction terms between each covariate and analysis time into the model to check the validity of the proportional hazards assumption. As the assumption was not met for some covariates (black/nonblack and education), we re-estimated Models 1-3 retaining these particular interactions. We found that results were not sensitive to their inclusion. All results incorporated sampling weights which capture unequal probabilities of selection and non-response adjustments and accounted for the complex survey design of NHANES. Analyses were conducted using STATA 12 (StataCorp, Texas, USA).

\section{Results}

Table 1 shows descriptive characteristics of the US adult population by diabetes status and period. In both periods, individuals in the dysglycemic groups were generally older, less educated, and more likely to be Hispanic or non-Hispanic black compared to those without diabetes. Changes over time in the distribution of smoking status and body mass index (BMI) differed across the three groups. The proportion of current smokers declined significantly over time for the pre-diabetic group (37.63\% to $22.13 \%$; $\mathrm{p}<0.05$ ), but not for the nondiabetic $(25.43 \%$ to $23.91 \%)$ and diabetic groups $(22.01 \%$ to $19.13 \%)$. Larger increases in the percentage of never smokers were observed in persons with dysglycemia compared to those without. The proportion with severe obesity (BMI $\geq 35 \mathrm{~kg} / \mathrm{m} 2$ ) increased in all groups, but somewhat larger absolute increases were observed in individuals with dysglycemia (differential increase of $5 \%$ [95\% CI $1-10 \% ; \mathrm{p}<0.05]$ ). The proportion with severe obesity was three times higher among individuals with diabetes than among those without diabetes in the more recent period. Table 1 also shows mean HbA1c levels, which remained essentially unchanged between periods for individuals with pre-diabetes and diabetes.

Table 2 shows age-standardized mortality rates and relative risks by diabetes status and period. Whereas the age-standardized mortality rate in the normo-glycemic population declined significantly between 1988-2001 and 1999-2006 (7.81 to 6.04; $\mathrm{p}$ for difference $<0.05$ ), absolute levels of mortality among individuals with prediabetes and diabetes showed no statistically significant change. The mortality rate increased from 11.19 to 14.02 deaths per 1,000 person-years in individuals with prediabetes and from 20.34 to 20.82 deaths per 1,000 person-years in individuals with diabetes. Relative risks of mortality grew across periods for both individuals with pre-diabetes (1.43 to 2.32) and those with diabetes (2.60 to 3.45). These changes were equivalent to increases of $62 \%$ and $32 \%$ in the relative risks and were not statistically significant.

The differences in mortality across the two periods in Table 2 may partially reflect changes in background factors related to both diabetes and mortality, such as race/ethnicity, education, smoking and BMI. Table 3 shows results from multivariate proportional hazards models adjusting for these covariates. In these models, the hazard ratio of the 'dysglycemia $\mathrm{x}$ period' term expresses the proportionate change in the hazard ratio associated with dysglycemia from the earlier to the later mortality period (1988-2001 to 1999-2006). In Model 1, adjusting for social and 
Table 1 Descriptive statistics of the U.S. adult population ages 35-74 by diabetes status and period

\begin{tabular}{|c|c|c|c|c|c|c|}
\hline & \multicolumn{2}{|c|}{ No Diabetes } & \multicolumn{2}{|c|}{ Pre-Diabetes } & \multicolumn{2}{|c|}{ Diabetes } \\
\hline & NHANES III & NHANES CTS & NHANES III & NHANES CTS & NHANES III & NHANES CTS \\
\hline Sample size & 6,277 & 4,014 & 1,679 & 660 & 1,254 & 796 \\
\hline Mean age, y & 49.45 & 49.47 & 56.19 & 55.52 & 57.11 & 56.99 \\
\hline Hispanic,\% & 7.62 & 10.84 & 10.26 & 15.65 & 11.25 & 16.91 \\
\hline Non-Hispanic Black,\% & 7.32 & 8.71 & 20.07 & 16.67 & 17.69 & 17.79 \\
\hline \multicolumn{7}{|l|}{ Education,\% } \\
\hline Less than High School & 20.99 & $17.66+$ & 35.63 & 29.06 & 44.35 & 37.61 \\
\hline High School & 33.21 & $24.45+$ & 35.56 & $28.52+$ & 33.15 & $24.84 \dagger$ \\
\hline More than High School & 45.80 & $57.89+$ & 28.82 & $42.42+$ & 22.51 & $37.55+$ \\
\hline \multicolumn{7}{|l|}{ Smoking Status, $\%$} \\
\hline Current & 25.43 & 23.91 & 37.63 & $22.13+$ & 22.01 & 19.13 \\
\hline Former & 31.51 & $28.51+$ & 30.04 & 33.85 & 40.98 & 34.56 \\
\hline Never & 43.06 & $47.58+$ & 32.33 & $44.02 \dagger$ & 37.02 & $46.31+$ \\
\hline Mean $B M l^{a}\left(k g / m^{2}\right)$ & 26.69 & $27.73+$ & 28.85 & $31.74+$ & 31.41 & $32.86+$ \\
\hline \multicolumn{7}{|l|}{ BMI $\left.\right|^{a}$ category $\left(\mathrm{kg} / \mathrm{m}^{2}\right), \%$} \\
\hline 30 to 35 & 15.09 & $17.41+$ & 20.34 & $31.98+$ & 28.25 & 25.68 \\
\hline More than 35 & 6.88 & $10.48+$ & 15.33 & $24.27+$ & 26.20 & $33.15+$ \\
\hline Mean HbA1c & 5.16 & $5.24+$ & 5.99 & 5.97 & 8.11 & 8.00 \\
\hline
\end{tabular}

BMI: body mass index; HbA1c: Hemoglobin A1c; a. BMI derived from measure data on height and weight; No Diabetes is defined as HbA1c less than 5.7\%; Pre-Diabetes is defined as HbA1c between 5.7 and 6.5\%; Diabetes is defined as HbA1c above $6.5 \%$ or on treatment for diabetes. Sources: National Health and Nutrition Examination Survey (NHANES) III (1988-1994) and continuos (CTS) (1999-2002).

† Significantly different $(\mathrm{p}<0.05)$ compared with NHANES III.

Table 2 All-cause mortality rates (per 1,000 person-years) and relative risks for the US adult population by diabetes status and period

\begin{tabular}{|c|c|c|}
\hline & $\begin{array}{l}\text { NHANES III } \\
(1988-2001)\end{array}$ & $\begin{array}{l}\text { NHANES CTS } \\
(1999-2006)\end{array}$ \\
\hline \multicolumn{3}{|l|}{ No Diabetes } \\
\hline Mortality Rate & $7.81(6.70-8.93)$ & $6.04(4.77-7.32)+$ \\
\hline Relative Risk & 1.00 & 1.00 \\
\hline \multicolumn{3}{|l|}{ Pre-Diabetes } \\
\hline Mortality Rate & 11.19 (8.38-14.00) & 14.02 (8.47-19.58) \\
\hline Relative Risk & $1.43(1.12-1.74)$ & $2.32(1.24-3.40)$ \\
\hline \multicolumn{3}{|l|}{ Diabetes } \\
\hline Mortality Rate & $20.34(16.00-24.67)$ & $20.82(13.10-28.54)$ \\
\hline Relative Risk & $2.60(1.91-3.29)$ & $3.45(2.02-4.87)$ \\
\hline Deaths & 960 & 341 \\
\hline Person-years & 67,526 & 30,989 \\
\hline
\end{tabular}

HbA1c: Hemoglobin A1c; No Diabetes is defined as HbA1c less than 5.7\%; PreDiabetes is defined as HbA1c between 5.7 and 6.5\%; Diabetes is defined as HbA1c above $6.5 \%$ or on treatment for diabetes. Sample includes persons ages 35-74 at baseline. Entry years are 1988-1994 with follow-up through 2001 for the earlier period and 1999-2002 with follow-up through 2006 for the later period. Mortality rates are age-standardized to the year 2000 Census population using age groups 35-54, 55-69 and 70-84. Relative risks are based on age-standardized mortality rates and are otherwise unadjusted. All estimates are weighted and account for complex survey design. Sources: National Health and Nutrition Examination Survey (NHANES) III and continuous (CTS).

${ }^{\dagger}$ Significantly different $(p<0.05)$ compared with NHANES III. demographic factors, the hazard ratio of dysglycemia was 1.63 (95\% CI: $1.38-1.92)$ in 1988-2001. The hazard ratio of the two-way interaction indicated that the relative risks increased by $34 \%$ across the two periods; however the difference was not significant. Multiplying these two terms yields a hazard ratio for dysglycemia of 2.18 (95\% CI: 1.573.04) in the later period (1999-2006).

When smoking was included in the model, we observed a statistically significant increase in the hazard ratio for dysglycemia across the two periods $(\mathrm{HR}=1.45$ for the interaction term; 95\% CI: 1.02-2.07). In Model 2, the hazard ratio of dysglycemia was 1.55 (95\% CI: 1.311.83) in 1988-2001 and 2.26 (95\% CI: 1.64-3.11) in 1999-2006. The increase in the magnitude of the interaction term between Model 1 and Model 2 was due to both a slight decline in the hazard ratio in the early period (from 1.63 to 1.55 ) and a slight increase in the hazard ratio applicable to the later period (2.18 to 2.26). These changes are likely attributable to the changing association between smoking and diabetes status across time as shown in Table 1. Additional adjustment for BMI and BMI-squared had little effect on the magnitude of the estimated change (Model 3).

\section{Discussion}

In this paper, we investigated mortality of individuals with dysglycemia relative to individuals with normo- 
Table 3 Hazard ratios predicting mortality from all causes

\begin{tabular}{|c|c|c|c|c|c|c|}
\hline & \multicolumn{2}{|c|}{ Model 1} & \multicolumn{2}{|c|}{ Model 2} & \multicolumn{2}{|c|}{ Model 3} \\
\hline & Hazard Ratio & $95 \% \mathrm{Cl}$ & Hazard Ratio & $95 \% \mathrm{Cl}$ & Hazard Ratio & $95 \% \mathrm{Cl}$ \\
\hline \multicolumn{7}{|l|}{ Sex } \\
\hline Women & 1.00 & & 1.00 & & 1.00 & \\
\hline Men & $1.54^{* * *}$ & $(1.34-1.76)$ & $1.30^{* * *}$ & $(1.13-1.50)$ & $1.33^{* * *}$ & $(1.16-1.53)$ \\
\hline \multicolumn{7}{|l|}{ Race/ethnicity } \\
\hline Other & 1.00 & & 1.00 & & 1.00 & \\
\hline Hispanic & 0.76 & $(0.57-1.02)$ & 0.83 & $(0.62-1.12)$ & 0.84 & $(0.63-1.13)$ \\
\hline Non-Hispanic Black & $1.48^{* * *}$ & $(1.21-1.82)$ & $1.51^{* * *}$ & $(1.23-1.84)$ & $1.49^{* * *}$ & $(1.22-1.81)$ \\
\hline \multicolumn{7}{|l|}{ Education Level } \\
\hline Less than High School & 1.00 & & 1.00 & & 1.00 & \\
\hline High School & $0.78^{* *}$ & $(0.65-0.93)$ & $0.79^{* *}$ & $(0.66-0.94)$ & $0.79^{* *}$ & $(0.67-0.94)$ \\
\hline More than High School & $0.61^{* * *}$ & $(0.50-0.74)$ & $0.68^{* * *}$ & $(0.56-0.83)$ & $0.67^{* * *}$ & $(0.55-0.82)$ \\
\hline \multicolumn{7}{|l|}{ Smoking } \\
\hline Never Smoker & & & 1.00 & & 1.00 & \\
\hline Former Smoker & & & $1.83^{* * *}$ & $(1.46-2.29)$ & $1.83^{* * *}$ & $(1.46-2.39)$ \\
\hline Current Smoker & & & $2.90^{* * *}$ & $(2.24-3.75)$ & $2.73^{* * *}$ & $(2.11-3.52)$ \\
\hline BMI & & & & & $0.87^{* * *}$ & $(0.82-0.93)$ \\
\hline BMI Squared & & & & & $1.00^{* * *}$ & $(1.00-1.00)$ \\
\hline Period $^{a}$ & 0.86 & $(0.65-1.13)$ & 0.86 & $(0.66-1.13)$ & 0.88 & $(0.67-1.15)$ \\
\hline Dysglycemia $^{b}$ & $1.63^{* * *}$ & $(1.38-1.92)$ & $1.55^{* * *}$ & $(1.31-1.83)$ & $1.62^{* * *}$ & $(1.36-1.93)$ \\
\hline Dysglycemia X Period & 1.34 & $(0.94-1.92)$ & $1.45^{*}$ & $(1.02-2.07)$ & $1.46^{*}$ & $(1.03-2.08)$ \\
\hline
\end{tabular}

a. Period is an indicator variable which takes a value of 1 in the later of the two periods in the analysis. Entry years are $1988-1994$ with follow-up through 2001 for the earlier period and 1999-2002 with follow-up through 2006 for the later period; b. Dysglycemia sample includes persons with HbA1c $\geq 5.7 \%$ or on treatment for diabetes. References categories for categorical variables indicated by HR =1.00; Sample includes persons ages 35-74 at baseline. All estimates are weighted and account for complex survey design.

Sources: National Health and Nutrition Examination Survey III and continuous. *** $\mathrm{p}<0.001 ;{ }^{* *}<0.01 ;{ }^{*} \mathrm{p}<0.05$

glycemia and the extent to which the level of excess mortality associated with dysglycemia, adjusting for confounding factors, has changed over the period 19882006. Whereas the age-standardized mortality rate declined significantly between periods in the normoglycemic population, mortality rates in individuals with pre-diabetes and diabetes showed no clear evidence of change. In our multivariate analysis, dysglycemia was associated with a non-significant increase in mortality over time in a model that adjusted for socio-demographic characteristics. However, a more pronounced and statistically significant increase in excess risk was observed upon further adjustment for smoking, suggesting that secular trends in smoking and its distribution by diabetes status may partially obscure change in the mortality risks of dysglycemia. Our multivariate results, based on adjustment for social and demographic variables, smoking and body mass index, indicated a $46 \%$ increase in the relative risk of dysglycemia for the US adult dysglycemic population.

Our findings follow on two previous nationally representative studies that have documented increasing gaps in mortality between individuals with and without diabetes. Excess mortality risks for those who reported a diabetes diagnosis were found to have increased over the 1970s and 1980s [13]. In a subsequent study that included more recent cohort experience from years 1988-1994 and mortality follow-up through 2001 excess mortality appeared to increase among women but not in men with diabetes relative to 1976-1980 [14].

Diabetes-related mortality trends have also been investigated using data from the Framingham Heart Study [29]. This study used clinically measured glucose levels and found that excess mortality declined between 1950-1979 and 1976-2005. However, in a sensitivity analysis in which the authors investigated trends in a more recent and narrow time interval (1971 to 2000), no statistically significant declines in absolute or excess allcause mortality were identified.

Our findings contrast with those of a recent study that investigated three-year US mortality rates across four cohorts (1997-1998, 1999-2000, 2001-2002 and 20032004) using data from the National Health Interview Survey [15]. This study found declines in excess 
mortality in individuals with previously diagnosed diabetes between 1997-1998 and 2003-2004. However, as one-third to one-fifth of the diabetic population is undiagnosed [19], trends identified in the prior study may not be reflective of those for the entire adult population with diabetes. Criteria for diagnosis and screening of diabetes changed over the period of the analysis, further complicating interpretation of trends based on selfreported data for defining diabetes. With respect to diagnostic criteria, in 1997 the ADA established new guidelines for the diagnosis of diabetes, based on FPG, which lowered the threshold of diagnosis from $140 \mathrm{mg} / \mathrm{dL}$ to $126 \mathrm{mg} / \mathrm{dL}$ [23]. Any changes in the severity distribution of diabetes induced by the change in guidelines, which are likely to have been implemented gradually over time, would have likely biased trends estimates using selfreported data. Secular increases in screening may have also affected the composition of the population with diabetes over the two periods, by increasing the proportion of diabetes cases detected early. However, in a sensitivity analysis, the findings of the prior study were found to be robust to the exclusion of individuals with recent onset diabetes (2 years or less) [15].

Our consideration of individuals with pre-diabetes as well as those with diabetes may have also contributed to the differences in findings between the two studies. Given the absence of population-based screening in the US, individuals with non-diabetic dysglycemia may have benefited less from health services than individuals with diabetes, leading to lower likelihoods of mortality improvements over time in that sub-population. Finally, differences between studies could relate to the fact that HbA1c may identify a somewhat different subset of individuals with diabetes than FPG and other diagnostic measures in common use [19].

Our results also differ with evidence from the UK, where inequalities in mortality between individuals with and without diabetes are reported to have declined in the interval 1996-2006 [30]. Health systems factors may partially account for these differences [31]. One study points to improved management of diabetes in the UK compared to the US [32].

A key strength of our analysis is our use of HbA1c criteria rather than self-report to identify individuals with dysglycemia. To our knowledge, this is the first study to use an objective blood marker in the examination of national diabetes-related mortality change in the US. An additional strength of our study is that we included adjustments for key confounding variables, such as obesity and smoking, in our multivariate analysis. Our analysis was limited by small sample sizes, which prevented an investigation of the role of specific causes of death or a more detailed exploration of the mechanisms underlying increased relative mortality risks between the two periods. For example, one factor that may have contributed is an increase in the incidence of diabetes among individuals with dysglycemia across the two time periods. Although we detected no significant differences in the extent of mortality change between persons with pre-diabetes vs. diabetes or in men vs. women, there may be sub-group heterogeneity that we could not discern given limited statistical power. Future research should explore mortality trends in specific population sub-groups defined by diabetes and sex, investigate trends across cohorts more narrowly defined in calendar years and explore the sensitivity of trends to the use of alternate diagnostic measures of diabetes, such as FPG. We cannot rule out the possibility that our findings are affected by residual confounding. For example, adjustment for BMI at baseline may not have fully captured patterns of change in the duration of obesity [33], central adiposity and other features of the metabolic syndrome.

In conclusion, we find no evidence that excess mortality in US adults with dysglycemia decreased over the interval 1988-2006. These findings were not explained by age, race or ethnicity, education, smoking or rising BMI levels in persons with dysglycemia. When contrasted with the long-term secular declines in allcause and cardiovascular mortality in the US population [34], the lack of improvement in mortality in the dysglycemic population is concerning and suggests that individuals with pre-diabetes and diabetes should be an important focus of future interventions aimed at improving population health in the US.

\section{Competing interests}

The authors declare that they have no competing interests.

\section{Authors' contributions}

AS and NKM conceived of the study's idea. AS compiled the data, conducted the statistical analysis and wrote the first draft of the paper. NKM contributed to the statistical analysis and writing of the manuscript. Both authors read and approved the final manuscript.

\section{Acknowledgements}

This project was supported by Grant Number R01AG040212 from the National Institute on Aging. The content is solely the responsibility of the authors and does not necessarily represent the official views of the National Institutes on Aging or the National Institutes of Health. We are grateful to Samuel Preston, Lucia Tiererova and the reviewers of this manuscript for their comments and suggestions.

\section{Author details}

${ }^{1}$ Population Studies Center, University of Pennsylvania, 3718 Locust Walk, 239 McNeil Building, Philadelphia, PA 19104-6298, USA. ${ }^{2}$ Department of Global Health, Emory University, 1518 Clifton Road, Atlanta, GA 30322, USA.

Received: 11 December 2011 Accepted: 31 January 2013

Published: 28 February 2013

\section{References}

1. Xu J, Kochanek KD, Murphy SL, Tejada-Vera B: Deaths: final data for 2007. Natl Vital Stat Rep 2010, 58. http://www.cdc.gov/nchs/data/nvsr/nvsr58/ nvsr58_19.pdf. 
2. Diabetes Prevention Program Research Group: Reduction in the incidence of type 2 diabetes with lifestyle intervention or metformin. N Engl J Med 2002, 346:393-403

3. UK Prospective Diabetes Study (UKPDS) Group: Intensive blood-glucose control with sulphonylureas or insulin compared with conventional treatment and risk of complications in patients with type 2 diabetes (UKPDS 33). Lancet 1998, 352:837-853.

4. UK Prospective Diabetes Study (UKPDS) Group: Tight blood pressure control and risk of macrovascular and microvascular complications in type 2 diabetes. BMJ 1998, 317:703-713.

5. The Scandinavian Simvastatin Survival Study Group: Cholesterol lowering with simvastatin improves prognosis of diabetic patients with coronary heart disease: a subgroup analysis of the Scandinavian simvastatin survival study (4S). Diabetes Care 1997, 20:614-620.

6. Gaede $\mathrm{P}$, Lund-Andersen $\mathrm{H}$, Parving $\mathrm{H}-\mathrm{H}$, Pedersen O: Effect of a multifactorial intervention on mortality in type 2 diabetes. $N$ Engl J Med 2008, 358:580-591.

7. Buse JB, Ginsberg HN, Bakris GL, Clark NG, Costa F, Eckel R, Fonseca V, Gerstein H, Grundy S, Nesto R, Pignone M, Plutzky J, Porte D, Redberg R, Stitzel K, Stone N: Primary prevention of cardiovascular diseases in people with diabetes mellitus: a scientific statement from the American heart association and the American diabetes association. Diabetes Care 2007, 30:162-172.

8. American Diabetes Association: Diagnosis and classification of diabetes mellitus. Diabetes Care 2010, 33(Suppl 1):S62-S69.

9. Nolte E, Bain C, McKee M: Diabetes as a tracer condition in international benchmarking of health systems. Diabetes Care 2006, 29:1007-1011.

10. Cowie CC, Rust KF, Ford ES, Eberhardt MS, Byrd-Holt DD, Li C, Williams DE, Gregg EW, Bainbridge KE, Saydah SH, Geiss LS: Full accounting of diabetes and pre-diabetes in the US population in 1988-1994 and 2005-2006. Diabetes Care 2009, 32:287-294.

11. Saydah SH, Fradkin J, Cowie CC: Poor control of risk factors for vascular disease among adults with previously diagnosed diabetes. JAMA 2004 291:335-342.

12. Saaddine JB, Cadwell B, Gregg EW, Engelgau MM, Vinicor F, Imperatore G, Narayan $\mathrm{V}$ : Improvements in diabetes processes of care and intermediate outcomes: United States, 1988-2002. Ann Intern Med 2006, 144:465-474.

13. Gu K, Cowie C, Harris M: Diabetes and decline in heart disease mortality in US adults. JAMA 1999, 282:1291-1297.

14. Gregg EW, Gu Q, Cheng YJ, Narayan KMV, Cowie CC: Mortality trends in men and women with diabetes, 1971 to 2000. Ann Intern Med 2007, 147:149-156.

15. Gregg EW, Cheng YJ, Saydah S, Cowie C, Garfield S, et al: Trends in death rates among U.S. Adults with and without diabetes between 1997 and 2006. Findings from the national health interview survey. Diabetes Care 2006, 2012(35):1252-1257.

16. Davidson J: Diagnosis of diabetes mellitus. In Clinical diabetes mellitus: A problem-oriented approach. Edited by Davidson J. New York: Thieme; 2000:169-188.

17. Gregg EW, Cadwell BL, Cheng YJ, Cowie CC, Williams DE, Geiss L, Engelgau $M M$, Vinicor F: Trends in the prevalence and ratio of diagnosed to undiagnosed diabetes according to obesity levels in the U.S. Diabetes Care 2004, 27:2806-2812.

18. Koopman R, Mainous A, Diaz V, Geesey M: Changes in age at diagnosis of type 2 diabetes mellitus in the United States, 1988 to 2000. Ann Fam Med 2005, 3:60-63.

19. Cowie CC, Rust KF, Byrd-Holt DD, Gregg EW, Ford ES, Geiss LS, Bainbridge KE, Fradkin JE: Prevalence of diabetes and high risk for diabetes using $\mathrm{A} 1 \mathrm{C}$ criteria in the U.S. population in 1988-2006. Diabetes Care 2010, 33:562-568

20. The National Health Interview Survey (1986-2004) Linked Mortality Files, mortality follow-up through 2006: Matching Methodology, May 2009. Hyattsville, MD: National Center for Health Statistics. Office of Analysis and Epidemiology; 2009.

21. Little RR, Wiedmeyer HM, England JD, Wilke AL, Rohlfing $\mathrm{CL}$, Wians FH Jr, Jacobson JM, Zellmer V, Goldstein DE: Interlaboratory standardization of measurements of glycohemoglobins. Clin Chem 1992, 38:2472-2478.

22. Kirkman MS, Kendall DM: Hemoglobin A1c to diagnose diabetes: why the controversy over adding a new tool? Clin Chem 2011, 57:255-257.

23. The Expert Committee on the Diagnosis and Classification of Diabetes Mellitus: Report of the expert committee on the diagnosis and classification of diabetes mellitus. Diabetes Care 1997, 26:5-20.
24. King G, Honaker J, Joseph A, Scheve K: Analyzing incomplete political science data: An alternative algorithm for multiple imputation. American Political Science Review 2001, 95:49-69.

25. Honaker J, King G, Blackwell M: AMELIA II: a program for missing data. http:// gking.harvard.edu/amelia/ (accessed September 3, 2012).

26. Kivimäki M, Ferrie JE, Batty GD, Davey Smith G, Elovainio M, Marmot MG, Shipley MJ: Optimal form of operationalizing BMI in relation to all-cause and cause-specific mortality: the original Whitehall study. Obesity 2008, 16:1926-1932

27. Wannamethee SG, Shaper a G, Perry IJ: Smoking as a modifiable risk factor for type 2 diabetes in middle-aged men. Diabetes Care 2001, 24:1590-1595.

28. Yeh HC, Duncan BB, Schmidt MI, Wang NY, Brancati FL: Smoking, smoking cessation, and risk for type 2 diabetes mellitus. Ann Intern Med 2010, 152:10-17.

29. Preis SR, Hwang S, Coady S, Pencina MJ, D'Agostino RB, Savage PJ, Levy D, Fox CS: Trends in all-cause and cardiovascular disease mortality among women and men with and without diabetes mellitus in the Framingham heart study, 1950 to 2005. Circulation 2009, 119:1728-1735.

30. Gulliford MC, Charlton J: Is relative mortality of type 2 diabetes mellitus decreasing? AJE 2008, 169:455-61.

31. Zhang X, Bullard KM, Gregg EW, Beckles GL, Williams DE, Barker LE, Albright $\mathrm{AL}$, Imperatore $\mathrm{G}$ : Access to health care and control of $\mathrm{ABCs}$ of diabetes. Diabetes Care 2012, 35:1566-71.

32. Mainous AG III, Diaz VA, Saxena S, Baker R, Everett CJ, Koopman RJ, Majeed A: Diabetes management in the USA and England: comparative analysis of national surveys. J R Soc Med 2006, 99:463-469.

33. Preston SH, Mehta NK, Stokes A: Modeling obesity histories in cohort analyses of health and mortality. Epidemiology 2013, 24:158-166.

34. Ford ES, Ajani UA, Croft JB, Critchley JA, Labarthe DR, Kottke TE, Giles WH, Capewell S: Explaining the decrease in U.S. deaths from coronary disease, 1980-2000. NEIM 2007, 356:2388-2398.

doi:10.1186/1478-7954-11-3

Cite this article as: Stokes and Mehta: Mortality and excess risk in US adults with pre-diabetes and diabetes: a comparison of two nationally representative cohorts, 1988-2006. Population Health Metrics 2013 11:3.

\section{Submit your next manuscript to BioMed Central and take full advantage of:}

- Convenient online submission

- Thorough peer review

- No space constraints or color figure charges

- Immediate publication on acceptance

- Inclusion in PubMed, CAS, Scopus and Google Scholar

- Research which is freely available for redistribution 